\title{
Optimized Query Evaluation Using Cooperative Sorts
}

\author{
Yu Cao, Ramadhana Bramandia, Chee-Yong Chan, Kian-Lee Tan \\ Department of Computer Science \\ National University of Singapore \\ \{caoyu, bramandia, chancy, tankl\}@comp.nus.edu.sg
}

\begin{abstract}
Many applications require sorting a table over multiple sort orders: generation of multiple reports from a table, evaluation of a complex query that involves multiple instances of a relation, and batch processing of a set of queries. In this paper, we study how multiple sortings of a table can be efficiently performed. We introduce a new evaluation technique, called cooperative sort, that exploits the relationships among the input set of sort orders to minimize $\mathrm{I} / \mathrm{O}$ operations for the collection of sort operations. To demonstrate the efficiency of the proposed scheme, we implemented it in PostgreSQL and evaluated its performance using both TPC-DS benchmark and synthetic data. Our experimental results show significant performance improvement over the traditional non-cooperative sorting scheme.
\end{abstract}

\section{INTRODUCTION}

Sorting is a frequently used and expensive operation in database systems. It is employed not only to produce sorted output, but also in many sort-based algorithms for aggregation, duplicate removal, join, and set operations. As such, it has been extensively studied [1], [2], [3], [4], [5], [6]. The standard technique adopted in most commercial systems is based on the external merge-sort algorithm.

In this paper, we investigate the problem of efficiently sorting a table in multiple sort orders. It turns out that such multiple sortings of a table is not uncommon in many applications. For example, in many organizations, many reports are generated at the end of the day/week/month. Typically, these reports contain the same content but in different sort orders. A bank may produce reports ordered by amount deposited/withdrawn/balance, date, branch, and so on. Similarly, examination schedules are usually printed in different orders - order by course number, dates, examiners, and invigilators. Yet another example arises in decision making applications where a complex query typically contains multiple instances of a base relation or view [7].

Now, in all these examples, the queries can be processed by sorting a table multiple times, once per sorting order. This seems to be wasteful of resources especially since we are sorting a single table (or materialized view) albeit over multiple sort orderings. Intuitively, if we can somehow salvage the (partial) work done to sort a table on a particular order for a subsequent sort order on the same table, then we may reduce the overall processing cost for multiple sortings. This is exactly what we set out to achieve in this paper.
We begin by considering the scenario of sorting a table $T$ in two different sort orders $o_{1}$ and $o_{2}$, where $o_{1}$ and $o_{2}$ are sequences of some attributes of $T$. Based on the relationships between $o_{1}$ and $o_{2}$, we identify four cases in which we can exploit the (partial) results of sorting $T$ on $o_{1}$ to speed up the sorting of $T$ on $O_{2}$. In case $1, O_{2}$ is a (strict) prefix of $o_{1}$, and in case 2, $o_{1}$ and $o_{2}$ share a common prefix. Under these situations, the sorted $T$ on $o_{1}$ can be re-used directly (for case 1) or via a light-weight reorganization (for case 2) to sort $T$ on $\mathrm{O}_{2}$. As a result, the cost to sort $\mathrm{T}$ on $\mathrm{O}_{2}$ is totally eliminated (case 1) or significantly reduced (case 2). We refer to these cases as result sharing of instance sorts.

While cases 1 and 2 are straightforward, cases 3 and 4 offer sharing opportunities that have not been previously explored. As an example of case 3, a prefix of $o_{2}$ appears as a substring of $o_{1}$, e.g., $o_{2}$ is the attribute $a_{2}$ and $o_{1}$ is a composite of two attributes $\left(a_{1}, a_{2}\right)$. In this case, the sorted $T$ on $o_{1}$ can be viewed as a concatenation of tuple clusters each of which contains records ordered on $o_{2}$. Thus, each tuple cluster of the sorted $T$ on $\left(a_{1}, a_{2}\right)$ corresponds to a distinct $a_{1}$ value. Within this cluster, the $a_{2}$ values are sorted. Thus, we could treat these tuple clusters as initial runs for the sort on $\mathrm{O}_{2}$, and reuse them to facilitate the run merging phase of the merge-sort algorithm without having to perform the (initial) run formation phase. However, a naive exploitation of these readily available runs may not be beneficial as there may be too many of such tuple clusters. To handle this, we propose a cooperative sort procedure. It first organizes tuples of $T$ into an intermediate form $T^{\prime}$ such that (a) $T^{\prime}$ can be used to produce sorted $T$ on $o_{1}$ efficiently with only (possibly) in-memory sorting; (b) $T^{\prime}$ can be used as initial runs for the sort on $o_{2}$ efficiently as $T^{\prime}$ is structured such that the total number of initial runs for sorting $\mathrm{T}$ on $\mathrm{O}_{2}$ has been significantly reduced (compared to that of using the tuple clusters from the sorted $T$ on $o_{1}$ ). Cooperative sort tries to keep as much the benefit of avoiding scanning $T$ and initial run formation for the sort on $o_{2}$ as possible by minimizing the cost of the corresponding run merging.

Finally, case 4 covers the most general scenarios where none of the prefixes of $o_{2}$ appear as substrings of $o_{1}$ and vice versa. As a simple example, we may want to sort $T$ on attribute $a_{1}$ as well as attribute $a_{2}$. This case can be easily handled by first sorting $T$ on $\left(a_{1}, a_{2}\right)$ and so reducing this case to case 3 .

When a table has to be sorted on more than two orders, we have further opportunity to optimize the performance. Given a 
table with multiple sorts, we model it as the minimum directed Steiner tree problem. As the number of sortings is manageable, we adopt a brute force algorithm to find the optimal solution on how each sort will be sequenced and completed.

Another direction that our work can be applied is in enriching the plan space for query optimization. For some query plan, it is possible that replacing a hash-join (in an optimal plan) with a sub-optimal sort-merge join can lead to an overall lower cost due to the sort-sharing opportunities either within the same query or among a batch of queries.

In this paper, we make the following key constributions: (1) We systematically study the problem of evaluating multiple sortings on a relation beginning with an analysis of the relationships for two sort orders and generalizing to multiple sort orders; (2) We propose a novel evaluation technique, cooperative sort, that optimizes the evaluation of two sort orders by deriving an intermediate hybird sort order; (3) We perform a comprehensive experimental evaluation of our proposed techniques with an implementation in PostgreSQL. Our performance results show that cooperative sort outperforms conventional sort on average by $25 \%$ and up to $35 \%$. Moreover, our study shows the potential benefit of enriching the optimizer search space with cooperative sort.

The rest of this paper is organized as follows. In Section II, we present some preliminaries. Section III formally discusses the techniques of result sharing and cooperation between two instance sorts. We elaborate the details of cooperative sort in Section IV. Further discussions about general sort sharing are presented in Section V. In Section VI, we generalize cooperative sort to evaluate more than two sort operations. Our experimental study presented in Section VII validates the effectiveness of our proposed techniques. We discuss relevant work in Section VIII and finally conclude in Section IX.

\section{PRELIMINARIES}

Sort orders are referred as $o, o_{1}, o_{2}$ etc., each of which is a sequence of distinct attributes $\left(a_{1}, a_{2}, \cdots a_{n}\right), n \geq 1$, of the relation $T^{1}$ to be sorted. In this paper we utilize the following main notations, some of which are borrowed from [8]:

- $s_{i}=\operatorname{sort}\left(T, o_{i}\right)$ : a sort operation $s_{i}$ on $T$, with order $o_{i}$.

- $\operatorname{cost}(s)$ : the I/O cost (in number of accessed blocks) for sort operation $s$.

- $\operatorname{attrs}(o)$ : the set of attributes in sort order $o$.

- $|o|$ : number of attributes in the sort order $o$.

- $o_{1}<o_{2}: o_{1}$ is a proper prefix of $o_{2}$.

- $o_{1} \leq o_{2}: o_{1}$ is a prefix of $o_{2}$.

- $o_{1} \wedge o_{2}$ : the longest common prefix between $o_{1}$ and $o_{2}$.

- $o_{1}+o_{2}$ : sort order obtained by concatenating $o_{1}$ and $o_{2}$.

- $o-A$ : sort order obtained by removing from $o$ those attributes that also appear in the set of attributes $A$.

- o-segment: the cluster of tuples in $T$ that have the same value for $\operatorname{attrs}(o)$.

- $B(e)$ : size of tuples of expression $e$, in number of blocks.

${ }^{1}$ For simplicity, our discussion assumes $T$ to be a relation, but our techniques also apply when $T$ is the output of some query subplan.
- $D(e, o)$ : number of distinct values for $\operatorname{attrs}(o)$ in tuples of expression $e$; i.e., $D(e, o)=\left|\pi_{o}(e)\right|$.

- $M$ : number of memory blocks available for sorting.

In this paper, we assume that initial sorted runs are generated using replacement selection, and our cost model assumes that each initial sorted run is of size $2 M$ blocks. The external sorting of a relation $T$ is done using the well-known $F$-way merge sort technique, where $F$ is the merge order (i.e., number of runs that can be merged using $M$ ). Our cost model for a sort operation $s$ on $T$ using $M$ blocks of memory is given by

$$
\operatorname{cost}(s)=2 \times B(T) \times\left(\left\lceil\log _{F}\left(\frac{B(T)}{2 M}\right)\right\rceil+1\right) .
$$

\section{SORT Sharing TECHNIQUeS}

In this section, we present an overview of techniques for optimizing the evaluation of multiple sorts on a relation $T$. We will first focus on the basic case involving only two sort operations, and then explain how our techniques can be easily extended to the general case in Section VI.

Consider two sort operations $s_{1}=\operatorname{sort}\left(T, o_{1}\right)$ and $s_{2}=$ $\operatorname{sort}\left(T, o_{2}\right)$. By exploiting the relationship between $o_{1}$ and $\mathrm{O}_{2}$, the total I/O cost of the two sortings can be reduced with one of two key techniques:

- Result sharing technique: the idea is to leverage the output of one sort operation to more efficiently evaluate the other sort operation.

- Cooperative sorting technique: the idea is to create "hybrid" sorted runs that can benefit the evaluation of both sort operations.

While the result sharing technique has been previously discussed in other contexts [9], [8], to the best of our knowledge, we are the first to investigate the cooperative sorting technique.

The relationships between $o_{1}$ and $o_{2}$ that we are interested in can be classified into four main cases:

- Case 1: $o_{2}$ is a prefix of $o_{1}$; i.e., $o_{2} \leq o_{1}$.

- Case 2: $o_{1}$ and $o_{2}$ share a non-empty common prefix which is a proper prefix of $o_{2}$; i.e, $0<\left|o_{1} \wedge o_{2}\right|<\left|o_{2}\right|$.

- Case 3: the set of attributes in $o_{2}$, where $o_{2}=o_{21}+o_{22}$, is a subset of the attributes in a prefix, $o_{11}+o_{12}$, of $o_{1}=o_{11}+o_{12}+o_{13}$, such that $o_{21}=o_{12}$, attrs $\left(o_{22}\right) \subseteq$ $\operatorname{attrs}\left(o_{11}\right),\left|o_{11}\right|>0,\left|o_{13}\right| \geq 0$ and $\left|o_{22}\right| \geq 0$.

- Case 4: $o_{1}$ and $o_{2}$ do not satisfy any of the above cases, but $o_{1}+\left(o_{2}-\operatorname{attrs}\left(o_{1}\right)\right)$ and $o_{2}$ satisfy Case 3 .

Example 3.1 Consider the following sort orders: $o_{1}=$ $\left(a_{1}, a_{2}\right), o_{2}=\left(a_{2}\right), o_{3}=\left(a_{1}, a_{3}, a_{2}\right), o_{4}=\left(a_{2}, a_{3}, a_{6}, a_{7}\right)$, and $o_{5}=\left(a_{6}, a_{3}, a_{2}\right)$. The pair $o_{1}$ and $o_{3}$ is an example of case 2 with a common prefix given by $\left(a_{1}\right)$. Two examples of case 3 are: the pair $o_{1}$ and $o_{2}$, and the pair $o_{4}$ and $o_{5}$. An example of case 4 is the pair $O_{3}$ and $O_{5}$.

The first two cases are the more familiar and simpler cases which can be efficiently evaluated using the result sharing technique. The last two cases are the two new scenarios that we investigate in this paper, and their evaluations can be optimized by our proposed cooperative sorting technique to be presented in the next section. Note that although case 3 seems rather 
contrived and specialized, this is actually the most fundamental case to optimize as the most general case 4 is evaluated by reducing it to case 3 .

In the remainder of this section, we discuss how to apply result sharing technique to evaluate case 1 and case 2 .

For the first case, since a relation $T$ sorted on $o_{1}$ is trivially also sorted on $o_{2}$, it is sufficient to perform only $\operatorname{sort}\left(T, o_{1}\right)$; therefore, $s_{2}$ is not evaluated explicitly and $\operatorname{cost}\left(s_{2}\right)=0$.

For case 2, suppose $o^{\prime}=o_{1} \wedge o_{2}$ such that $o_{1}=o^{\prime}+o_{1}^{\prime}$, $o_{2}=o^{\prime}+o_{2}^{\prime},\left|o_{1}^{\prime}\right| \geq 0$ and $\left|o_{2}^{\prime}\right|>0$. In this case, a relation $T$ sorted on $o_{1}$ is also partially sorted on $o_{2}$ : the output of $s_{1}$ can be viewed as a concatenation of $o^{\prime}$-segments, and each such segment can be sorted independently on $o_{2}^{\prime}$ to form the sorted output for $s_{2}$. If the size of each $o^{\prime}$-segment is no larger than $M$ blocks, then the sorting of each segment on $o_{2}^{\prime}$ can be performed efficiently using internal sorting and $s_{2}$ can be evaluated with only a single pass of reading the output of $s_{1}$. As noted by [8], the strategy to evaluate $s_{2}$ by sorting $o^{\prime}$-segments also helps to significantly reduce the number of tuple comparisons: the complexity of independently sorting $k$ segments each of size $n / k$ tuples is $O(k * n / k \log (n / k))=$ $O(n \log (n / k))$ in contrast to a complexity of $O(n \log (n))$ for a single sort of all $n$ tuples.

For case $2, s_{1}$ is evaluated using conventional external merge-sort and $\operatorname{cost}\left(s_{1}\right)$ is given by Equation 1. Following [8], $\operatorname{cost}\left(s_{2}\right)=\sum_{i=1}^{D\left(T, o^{\prime}\right)} \operatorname{cost}\left(\operatorname{sort}\left(s e_{i}, o_{2}^{\prime}\right)\right)$, where $\operatorname{cost}\left(\operatorname{sort}\left(s e_{i}, o_{2}^{\prime}\right)\right)$ denotes the cost of sorting the $i$ th $o^{\prime}-$ segment $s e_{i}$ in the sorted output of $s_{1}$. If $B\left(s e_{i}\right) \leq M$, $\operatorname{cost}\left(\operatorname{sort}\left(s e_{i}, o_{2}^{\prime}\right)\right)$ is simply the cost of performing an internal sorting; otherwise, it is given by Equation 1. If we assume that the values of $o^{\prime}$ follow a uniform distribution, then $B\left(s e_{i}\right)=$ $B(T) / D\left(T, o^{\prime}\right)$.

\section{CoOperative Sorting}

In this section, we present a novel technique, termed cooperative sorting, to efficiently evaluate two sort operations $s_{1}=\operatorname{sort}\left(T, o_{1}\right)$ and $s_{2}=\operatorname{sort}\left(T, o_{2}\right)$, when $o_{1}$ and $o_{2}$ satisfy cases 3 or 4 identified in the previous section. For simplicity, we assume in this section that all the attributes in a sort order are to be sorted in ascending order. We discuss how to handle a combination of ascending and descending sorting orders in Section $\mathrm{V}$ and how to optimize evaluation for more than two sort operations in Section VI.

We first explain how the most general case 4 can be evaluated by reducing the problem to case 3 . The reduction is achieved by defining a new sort order $o_{1}^{\prime}=o_{1}+\left(o_{2}-\right.$ $\left.\operatorname{attrs}\left(o_{1}\right)\right)$. Now, the pair of sort orders $o_{1}^{\prime}$ and $o_{2}$ satisfy case 3 (by definition), and the sort operations $s_{1}$ and $s_{2}$ can be computed indirectly by evaluating the pair of sort operations on orders $o_{1}^{\prime}$ and $o_{2}$. Since $o_{1}$ is a proper prefix of $o_{1}^{\prime}$, the sorted output on $o_{1}^{\prime}$ is also sorted on $o_{1}$.

In the rest of this section, we turn our attention to the evaluation of case 3 using cooperative sorting.

\section{A. Key Ideas}

Recall that for case 3, we have $o_{1}=o_{11}+o_{12}+o_{13}$ and $o_{2}=o_{21}+o_{22}$, such that $o_{12}=o_{21}$, $\operatorname{attrs}\left(o_{22}\right) \subseteq \operatorname{attrs}\left(o_{11}\right)$, $\left|o_{11}\right|>0,\left|o_{13}\right| \geq 0$ and $\left|o_{22}\right| \geq 0$.

Observe that the output of $s_{1}$ can be viewed as a concatenation of $o_{11}$-segments, each of which is also sorted on $o_{2}$ and can be used as an initial sorted run for $s_{2}$. Thus, the result sharing technique can actually be applied to evaluate case 3 by first evaluating $s_{1}$ followed by merging the resulting $o_{11^{-}}$ segments to compute $s_{2}$. However, depending on $D\left(T, o_{11}\right)$ and data skew, the number of $o_{11}$-segments generated by $s_{1}$ could be very large with many small segments. In this situation, merging a large number of small sorted runs to evaluate $s_{2}$ could lead to an overall performance that is bad or even worse than performing a conventional external sorting of $\mathrm{T}$ on $\mathrm{O}_{2}$. The following example illustrates the limitation of the result sharing technique.

Example 4.1 Consider the relation $T(a, b)$ in Fig. 1, which is used as a running example in this section. Assume the following: each tuple occupies one disk block, the available sorting memory can hold four tuples (i.e., $M=4$ ), and the merge order $F=2$. Consider two sort operations $s_{1}$ and $s_{2}$ on $T$, with orders $o_{1}=(a, b)$ and $o_{2}=(b)$, respectively. Obviously, $o_{1}$ and $o_{2}$ satisfy case 3 with $o_{11}=(a)$. The output of $s_{1}$ is a concatenation of six a-segments ( $s e_{1}$ to $s e_{6}$ ), each of which is sorted on $(b)$. By applying the result sharing technique to evaluate $s_{2}$, the six $a$-segments of $s_{1}$ can be used as initial sorted runs for $s_{2}$ and merged to evaluate $s_{2}$ using three merge passes with $F=2$. However, this result sharing is actually more costly than a conventional external sorting: using replacement selection, three initial sorted runs are generated which can be merged with only two merge passes.

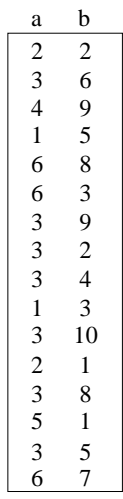

relation $\mathrm{T}$

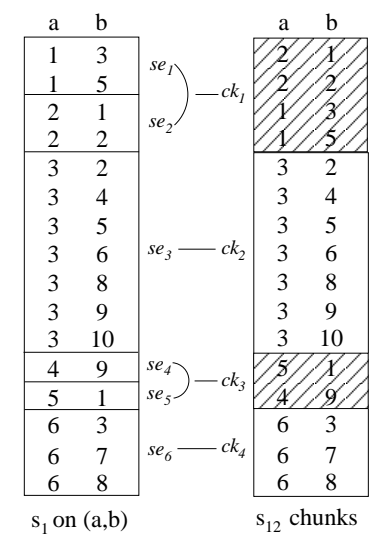

$$
\mathrm{s}_{1} \text { on }(\mathrm{a}, \mathrm{b})
$$

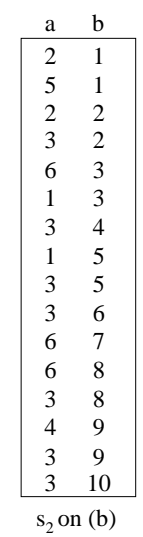

$F=2$
To avoid the drawback of result sharing technique, we propose a novel technique, cooperative sorting, that derives the outputs of both $s_{1}$ and $s_{2}$ from the output of an intermediate sort operation $s_{12}$ that is based on a "hybrid" sort order. The goal is to take into account the available sorting memory $M$ to generate longer (and thus fewer) initial sorted runs to reduce the cost of run merging phase for $s_{2}$.

The key idea behind cooperative sorting is the use of an intermediate sort operation $s_{12}$ that is based on a novel hybrid 
sort order. The output of $s_{12}$ is a sequence of chunks of tuples which are classified into natural chunks and composite chunks such that the tuples in the natural chunks are ordered by $o_{1}$ and the tuples in the composite chunks are ordered by $o_{2}$. The chunks are defined in terms of the $o_{11}$-segments in the output of $s_{1}$ as follows. Conceptually, the output of $s_{1}$ is a sequence of $o_{11}$-segments which can be partitioned into a sequence of chunks each of which consists of a consecutive sequence of $o_{11}$-segments. A composite chunk consists of two or more consecutive $o_{11}$-segments such that (1) the size of the chunk in terms of the total number of tuples is at most $M$, and (2) the tuples in the chunk are sorted on $o_{2}$. A natural chunk consists of exactly one $o_{11}$-segment and the tuples are sorted on $o_{1}$; there is no constraint on the size of a natural chunk.

Example 4.2 Consider again the running example in Fig. 1. Based on $o_{1}=(a, b)$ and $o_{2}=(b)$, the output of $s_{1}$, which is partitioned into six $a$-segments ( $s e_{1}$ to $s e_{6}$ ), can be organized into four $s_{12}$ chunks consisting of two composite chunks, $c k_{1}$ and $c k_{3}$ (shown shaded), and two natural chunks, $c k_{2}$ and $c k_{4}$ (shown non-shaded). The size of each composite chunk is at most $M$ (i.e., 4 tuples).

The chunks in $s_{12}$ are defined to satisfy the following three useful properties. First, the chunks are $o_{1}$-order preserving in the sense that if a chunk $c k_{i}$ precedes another chunk $c k_{j}$ in the output of $s_{12}$, then every tuple in $c k_{i}$ has a smaller $o_{1}$ value than every tuple in $c k_{j}$. Second, since the size of each composite chunk is no more than $M$, which is the size of the sorting memory, the tuples in a composite chunk can be sorted very efficiently using internal sorting. The above two properties enable $s_{1}$ to be efficiently derived from $s_{12}$ by a sequential scan of the chunks. Third, the tuples in each chunk of $s_{12}$ are sorted on $\mathrm{O}_{2}$.

We now elaborate on how both $s_{1}$ and $s_{2}$ are derived from the output of $s_{12}$. To derive $s_{1}$, the $s_{12}$ chunks are scanned and processed sequentially: if the chunk is a natural chunk, the tuples are already ordered on $o_{1}$ and can simply be output sequentially; otherwise, we first load all the tuples in the chunk into the sorting memory, internally sort the tuples on $o_{1}$, and then output the sorted tuples sequentially. To derive $s_{2}$ from $s_{12}$, we treat each chunk as an initial sorted run for $s_{2}$ (due to third property) and merge these chunks to derive $s_{2}$.

Since the derivation of $s_{2}$ from $s_{12}$ is more efficient if there are fewer chunks to be merged, the composite $s_{12}$ chunks should be constructed as large as possible (within the size constraint) to reduce the number of $s_{12}$ chunks. The minimum number of $s_{12}$ chunks can be generated efficiently by a greedy heuristic that sequentially scans the $o_{11}$-segments of $s_{1}$ as follows: if the size of a segment $s e_{i}$ exceeds $M$, then $s e_{i}$ forms a natural segment; otherwise, determine the longest sequence of consecutive segments $s e_{i}, s e_{i+1}, \cdots s e_{j}$ such that its total size is no more than $M$ tuples. If $i=j$, then $s e_{i}$ forms a natural segment; otherwise, create a composite chunk with the segments $s e_{i}, \cdots, s e_{j}$.

By deriving both $s_{1}$ and $s_{2}$ from the $s_{12}$ chunks, cooperative sorting avoids the $\mathrm{I} / \mathrm{O}$ cost of independently generating two sets of initial sorted runs for $s_{1}$ and $s_{2}$. However, there are three potential sources of overhead for cooperative sorting. First, the $s_{12}$ chunks are actually created by merging the initial sorted runs of $s_{1}$ (to be explained in the next subsection), which requires a non-trivial extension of the conventional run merging technique. Thus, the total cost of computing $s_{12}$ chunks could be more costly than computing $s_{1}$. Second, the number of $s_{12}$ chunks generated by cooperative sorting could still be more than the number of initial sorted runs for $s_{2}$ generated by the conventional initial run formation phase, possibly resulting in a more costly run merging phase for cooperative sorting. Third, the derivation of $s_{1}$ from $s_{12}$ incurs the computation overhead of performing internal sorts on composite chunks. Therefore, cooperative sorting is not always superior to conventional sorting. Thus, both approaches should be considered in a cost-based manner by the query optimizer for evaluating multiple sorts on a relation.

\section{B. Overview of Hybrid Sort Operation}

In this section, we present an overview of our proposed approach to perform $s_{12}$, the intermediate sort operation whose output is used to derive $s_{1}$ and $s_{2}$. The computation of $s_{12}$ consists of four main steps. In the first step, we scan the relation $T$ to create initial $s_{1}$ runs (i.e., initial sorted runs on $o_{1}$ ). This step is performed using the conventional run formation technique. During the first step, we also collect the following information about $T$ and the initial $s_{1}$ runs: the number of distinct $o_{11}$ values in $T$, and the number of tuples corresponding to each distinct $o_{11}$ value on each initial $s_{1}$ run. Thus, at the end of the first step, we know the size of each $o_{11}$-segment and the distribution of each $o_{11}$-segment's tuples among the initial $s_{1}$ runs.

In the second step, based on the information collected from the first step, we apply the previously described greedy algorithm to determine a partitioning of the sequence of $o_{11^{-}}$ segements in $T$ into a minimum number of $s_{12}$ chunks. This partitioning provides the following information about the output of $s_{12}$ : the number and the sequence of $s_{12}$ chunks, the size of each chunk, and the $o_{11}$-segments that comprise each chunk. Note that the tuples that belong to the same $s_{12}$ chunk are generally distributed across several initial $s_{1}$ runs. Since the $s_{12}$ chunks are $o_{1}$-order preserving, each initial $s_{1}$ run is actually partitioned into a sequence of chunklets, where each chunklet is the subset of tuples in the $s_{1}$ run that belong to the same $s_{12}$ chunk. We use the notation $c k l_{i, j}$ to denote the chunklet in the $j^{\text {th }}$ initial $s_{1}$ run that belongs to the $i^{\text {th }}$ chunk in $s_{12}$. Similar to chunks, chunklets are classified into natural and composite chunklets.

Example 4.3 Fig. 2 illustrates the two initial $s_{1}$ runs (ordered on $o_{1}=a$ ) generated from the relation $T$ in our running example in Fig. 1. The first initial run consists of chunklets $c k l_{1,1}, c k l_{2,1}, c k l_{3,1}$, and $c k l_{4,1}$, while the second initial run consists of chunklets $c k l_{1,2}, c k l_{2,2}, c k l_{3,2}$, and $c k l_{4,2}$.

In the third step, we merge the initial $s_{1}$ runs to generate the initial $s_{12}$ runs. Essentially, each initial $s_{12}$ run is created 

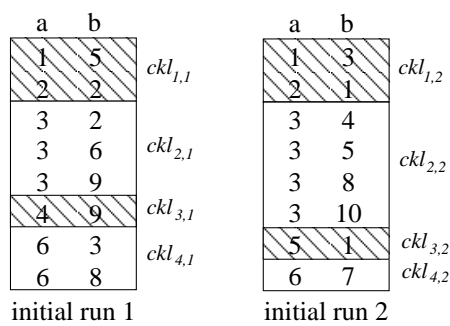

Fig. 2. Initial $s_{1}$ Runs for Relation $T$ in Example of Fig. 1

by merging a set of $F$ initial $s_{1}$ runs; specifically, the corresponding chunklets in the $F$ input $s_{1}$ runs are merged to form a longer chunklet in the output $s_{12}$ run. Thus, each initial $s_{12}$ run is also a sequence of $s_{12}$ chunklets, and the chunklets in the initial $s_{12}$ runs satisfy the same three properties as $s_{12}$ chunks. This merging operation is more complex than the conventional run merging step and will be elaborated in Section IV-C.

In the fourth step, the initial $s_{12}$ sorted runs are merged to generate the output of $s_{12}$. This is done using the conventional external run merging technique with a minor extension for the tuple comparison operator to take into account the different sorting orders of tuples within natural and composite chunks. Specifically, when comparing two tuples $t_{1}$ and $t_{2}$ during the merging, if $t_{1}$ and $t_{2}$ belong to the same composite (resp. natural) chunk, then $t_{1}$ precedes $t_{2}$ iff $t_{1}$ has a smaller value for $o_{2}$ (resp. $o_{1}$ ) compared to $t_{2}$; otherwise, $t_{1}$ precedes $t_{2}$ iff $t_{1}$ belongs to a chunk that precedes $t_{2}$ 's chunk in $s_{12}$. To facilitate the subsequent merging of $s_{12}$ chunks to derive $s_{2}$, the final pass of this merging step organizes the final single $s_{12}$ run into separate $s_{12}$ chunks. Note that the fourth step is skipped if the the third step produces only one initial $s_{12}$ run.

\section{Generating Initial $s_{12}$ Runs}

In this section, we elaborate on the procedure to merge initial $s_{1}$ runs to generate initial $s_{12}$ runs. Unlike the run merging procedure in conventional external sorting algorithm, this procedure in cooperative sorting is more intricate due to the fact that the input and output runs in our merge operation are of different "types": we are merging input runs that are ordered on $o_{1}$ to generate longer output runs that that sorted on the hybrid order for $s_{12}$ defined in Section IV-A. For the case where the chunklets in the input runs to be merged are natural chunklets, the merging procedure is simple and follows the conventional external merge procedure since the input and output orders are the same. However, for the case where the chunklets to be merged are composite chunklets, the merging requires an internal sorting operation to order the tuples in the merged chunklet on $o_{2}$. This requires loading into memory all the chunklet tuples that belong to the same chunk for sorting. In this section, we propose an efficient batched reading strategy to judiciously load tuples from the sorted runs into the sorting memory to optimize the I/O performance.

To motivate the need for our proposed scheme, consider the following simple conservative approach to merge $F$ initial $s_{1}$ runs into an initial $s_{12}$ run. The merging processes the chunklets in the input sorted runs one chunk at a time based on the chunk order. If the current chunk being processed is composite, we first read all the tuples in the chunklets from the $F$ input runs that belong to this chunk into the sorting memory, perform an internal sorting of the tuples, and output the merged chunklet. If the current chunk being processed is natural, we read $n$ tuples from the corresponding chunklet of each input run, where $n=\min \{$ size of the chunklet, $\lfloor M / F\rfloor\}$, and merge them using the conventional merging technique. While this conservative approach is sound, this approach might be fragmenting the reading of an input run into too many short sequential I/O reads. On the other hand, an arbitrary tuple reading strategy can lead to "deadlock" situations. For example, consider the two initial $s_{1}$ runs shown in Fig. 1 and suppose that we are merging chunklets for the first chunk with $M=4$. If we had read in three tuples from the first initial $s_{1}$ run into the sorting memory, then a deadlock situation would arise as the remaining sorting memory space is not adequate to permit loading in all the tuples from the first chunklet of the second initial $s_{1}$ run for sorting. Thus, the goal of our proposed batched reading strategy is to decompose the reading of each input run into as few batched reads of the tuples as possible to maximize sequential $\mathrm{I} / \mathrm{O}$ without getting into deadlocks.

Our proposed batched read strategy consists of two main steps. First, we partition each initial $s_{1}$ run into a sequence of batches, where each batch corresponds a sequence of tuples in the run. A run that has been partitioned in $n$ batches will be read into the sorting memory using $n$ read requests, each time reading in a complete batch of tuples. The second step then merges the initial $s_{1}$ runs based on the schedule of batched reads produced by the first step.

Since the merging of composite chunklets require an internal sorting, all the chunklets belonging to the same composite chunk among the $F$ runs being merged need to fit into the available sorting memory $M$ along with any previously read tuples that have yet to be merged. Let $R P_{i}^{m}$ (resp. $R P_{i}^{d}$ ) denote the set of tuples that belong to the chunk $c k_{i}$ that are in the sorting memory (resp. still on disk). Thus, for any composite chunk $c k_{i}$ that has some tuple in the sorting memory which has yet to be merged, $c k_{i}$ must satisfy the following constraint to avoid the previously described deadlock problem:

$$
B\left(R P_{i}^{m}\right)+B\left(R P_{i}^{d}\right)+\sum_{k>i} B\left(R P_{k}^{m}\right) \leq M
$$

We classify a chunk/chunklet as a small chunk/chunket if its size is no larger than $\lfloor M / F\rfloor$; otherwise, it is considered to be a large chunk/chunket.

For simplicity, our batched read strategy is designed based on the following two rules:

- Each composite chunklet, as well as each small natural chunklet will be completely read in one batched read.

- Each large natural chunklet will be read by a series of batched reads each with size of $\lfloor M / F\rfloor$ followed by a final batched read. Moreover, the first batched read will start from the head of the chunklet.

It follows that each batched read can be classified into one of four types based on its starting and ending points:

1) the batch starts from the head of a composite/natural chunklet and ends at the tail of a composite/natural 
chunklet.

2) the batch starts from the head of a natural chunklet and ends inside the same chunklet.

3) the batch starts and ends inside a natural chunklet.

4) the batch starts inside a natural chunklet and ends at the tail of a composite/natural chunklet.

For both type- 2 and type- 3 batch reads, their sizes are $\lfloor M / F\rfloor$.

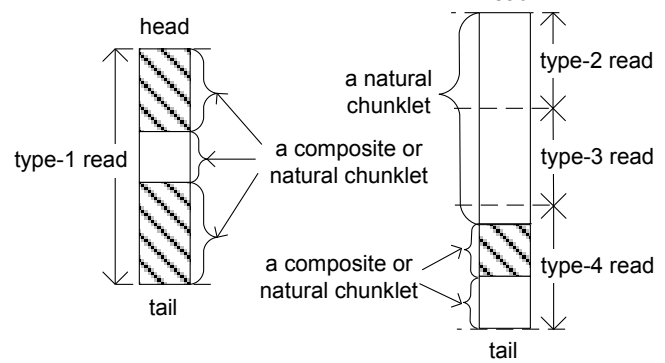

Fig. 3. Illustration of Four Types of Batched Reads

Fig. 3 illustrates the four types of batch reads. A large natural chunklet is read by batched reads of types 2, 3 and 4, while a composite or small natural chunklet is read by batched reads of types 1 and 4 . Each type- 2 read corresponds to a type4 read and a (possibly empty) set of type-3 reads.

Moreover, to balance the reading opportunity among natural chunklets among the runs being merged, we make two further restrictions: (1) in a type-1 read, the total size of a natural chunklet along with any following tuples must not exceed $\lfloor M / F\rfloor$; (2) the size of a type-4 read is at most $\lfloor M / F\rfloor$.

For the $F$ initial $s_{1}$ runs, Algorithm 1 precomputes a sequence $B R$ of all type- 1 , type- 2 and type- 4 reads to be conducted during the actual merging. The composition of each type- 1 or type- 4 read is decided using Algorithm 2. Note that the start point of a type-4 read is fixed within a natural chunklet. $B R$ does not record type-3 reads as they are fixed and can be easily deduced from each pair of type- 2 and type4 reads. Based on $B R$, Algorithm 3 performs the merging of $s_{1}$ runs by reading each run in batches. Note that although the runtime merging process follows the static schedule $B R$ strictly for type- 1 and type- 2 reads, the actual ordering for type- 3 and type- 4 reads for natural chunklets might need to be determined dynamically at runtime for correctness. More specifically, a type- 3 or type- 4 batch read of a run will be dynamically selected as the next read to be executed if the set of in-memory tuples $S$ of the most-recently read batch from this run belong to a natural chunk and $S$ will be earliest batch of in-memory tuples to be exhausted by the merge.
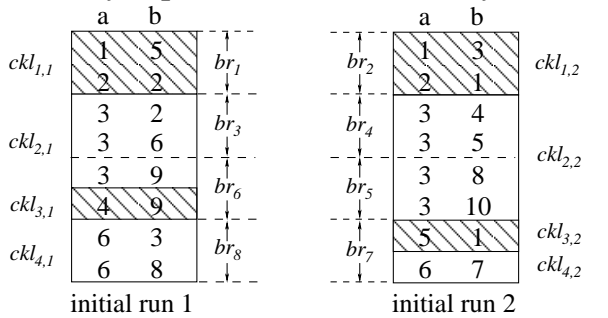

$$
\begin{aligned}
& M=4 \\
& F=2
\end{aligned}
$$

Fig. 4. Batched Read Sequence for Merging Two Initial $s_{1}$ Runs in Fig. 2

Fig. 4 shows the sequence of eight batched reads ( $b r_{1}$ to $b r_{8}$ ) conducted during the merging of the two initial $s_{1}$ runs in Fig. 2. $b r_{1}, b r_{2}, b r_{7}$ and $b r_{8}$ are type-1 reads; $b r_{3}$ and $b r_{4}$ are type- 2 reads, while $b r_{6}$ and $b r_{5}$ are their corresponding type4 reads respectively. There is no type- 3 read in this example. Table I shows the calculated $B R$. Note that since the last tuple $(3,5)$ read by $b r_{4}$ is smaller than the last tuple $(3,6)$ read by $b r_{3}$, tuples read by $b r_{4}$ will be exhausted first and thus $b r_{5}$ is actually read before $b r_{6}$ at runtime, which cannot be predicted according to $B R$.

TABLE I

The ENTRIES IN $B R$ FOR EXAMPLE IN FIG. 4

\begin{tabular}{|c|c|c|c|c|c|c|c|c|}
\hline$i$ & 1 & 2 & 3 & 4 & 5 & 6 & 7 & 8 \\
\hline$B R[i]$ & $b r_{1}$ & $b r_{2}$ & $b r_{3}$ & $b r_{6}$ & $b r_{4}$ & $b r_{5}$ & $b r_{7}$ & $b r_{8}$ \\
\hline
\end{tabular}
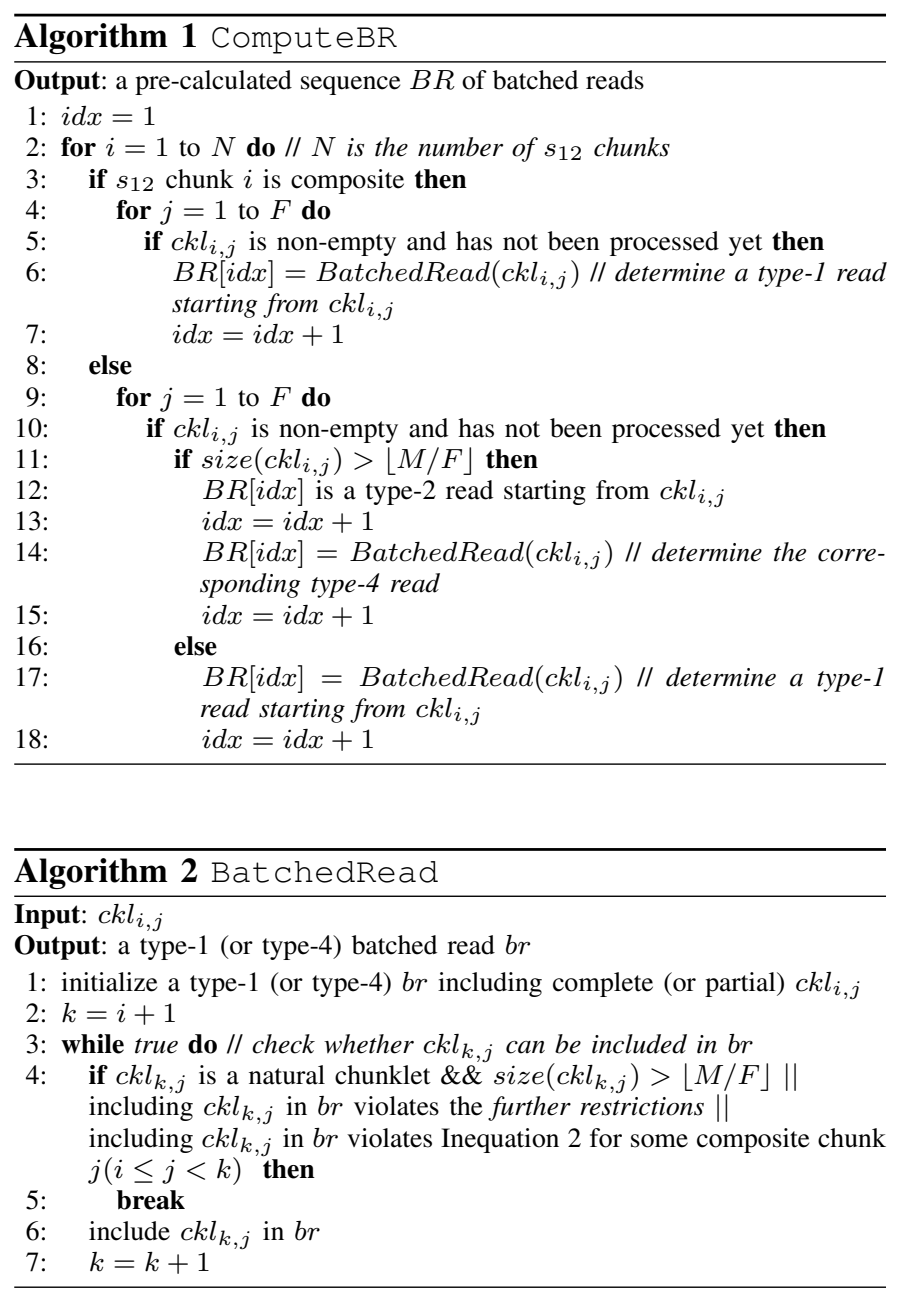

\section{Cost Model}

In this section, we present a simple analytical cost model for cooperative sorting. The total cost of using a cooperative sort $s_{12}$ to evaluate two sort operations $s_{1}$ and $s_{2}$ consists of three components: (1) the cost $C_{s_{12}}$ of generating $s_{12}$ chunks, which is assumed to be equal to the cost $C_{s_{1}}$ incurred for evaluating $s_{1}$ (given by Equation 1), plus the cost $C_{i s}$ of performing internal sortings on composite chunklets within initial $s_{1}$ runs; (2) the cost $C_{s_{12} \rightarrow s_{1}}$ of deriving $s_{1}$ is equal to the total cost of performing internal sortings for all the composite $s_{12}$ chunks; 


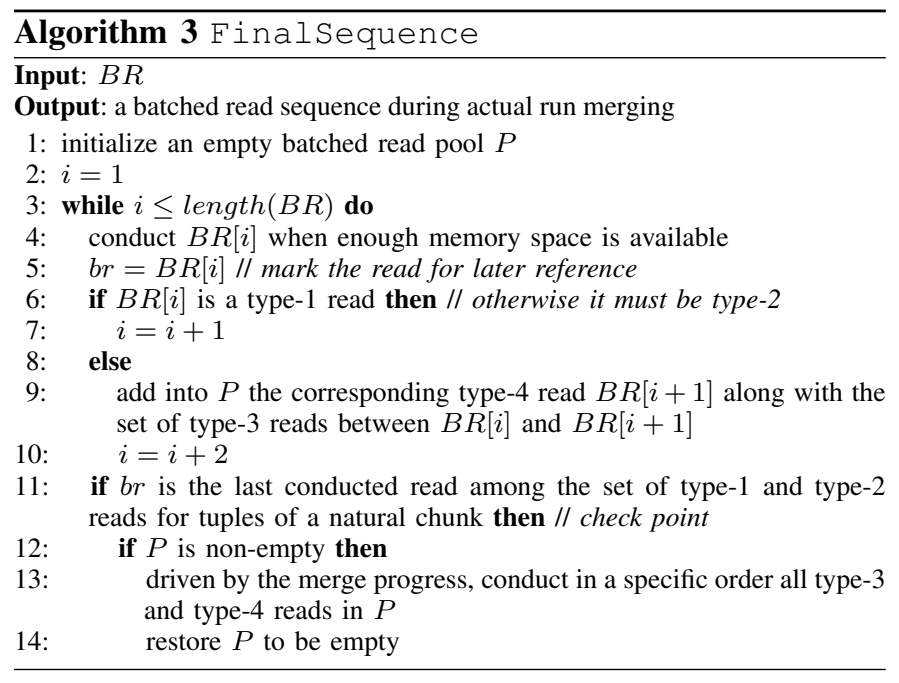

(3) the cost $C_{s_{12} \rightarrow s_{2}}$ of deriving $s_{2}$ is given by $2 \times B(T) \times$ $\left\lceil\log _{F} N\right\rceil$, where $N$ is the number of $s_{12}$ chunks.

Assuming a uniform distribution for the values of $o_{11}$, there are two cases to be considered.

Case 1: $B(T) / D\left(T, o_{11}\right) \leq 0.5 M$. All $s_{12}$ chunks are composite, and

$$
N=\left\lceil D\left(T, o_{11}\right) / k\right\rceil
$$

where $k$ is the maximal integer such that $k \times$ $B(T) / D\left(T, o_{11}\right) \leq M$.

Let $c p u \_c o s t(S)$ denote the cost of internally sorting tuples of total size $S$. We have

$$
\begin{aligned}
C_{i s} & =\frac{B(T)}{2 M} \times N \times \text { cpu_cost }(2 M / N) \\
C_{s_{12} \rightarrow s_{1}} & =N \times \text { cpu_cost }\left(k \times B(T) / D\left(T, o_{11}\right)\right)
\end{aligned}
$$

Case 2: $B(T) / D\left(T, o_{11}\right)>0.5 M$. All $s_{12}$ chunks are natural, and

$$
\begin{aligned}
N & =D\left(T, o_{11}\right) \\
C_{i s} & =C_{s_{12} \rightarrow s_{1}}=0
\end{aligned}
$$

Note that in the second case, the total cost of cooperative sorting is actually the same as that of applying result sharing technique. The performance of cooperative sorting depends partially on $D\left(T, o_{11}\right)$ and the relative sizes of $o_{11}$-segments. Besides the distinct value cardinality of $o_{11}$, the statistical value distribution of $o_{11}$ has little impact on the performance.

\section{E. Extensions}

In this section, we describe two important extensions of cooperative sorting.

1) Final Merge Optimization: If the external sorting operation is part of a pipelining query plan, a common optimization is to stop the run merge phase just before the final merge step so that the final merge step can be done as part of the generation of the sorted output. In this way, the final merge optimization saves one read and one write scan on $T$.

If the final merge optimization is enabled, the run merging phase of cooperative sorting will end up with $N(1<N \leq F)$ $s_{12}$ runs. The output of $s_{1}$ is derived by merging the $N s_{12}$ runs on-the-fly, with the batched reading strategy being used to sort the tuples in composite chunklets based on $o_{1}$ before the merging. As for $s_{2}$, each chunklet within the $s_{12}$ runs is treated as an initial run for $s_{2}$. For the special case where the number of initial $s_{1}$ runs generated for $s_{12}$ is no more than $F$, these initial $s_{1}$ runs can be transformed into initial $s_{2}$ runs by simply sorting the composite chunklets based on $o_{2}$.

2) Adapting to Other Merge Patterns: Our description of cooperative sorting in Section IV-B has assumed that the sorted runs are merged using $k$-way merge pattern for ease of presentation. The cooperative sorting approach can be easily adapted to other merge patterns such as polyphase merge and cascade merge [1]. In the general case, the collection of the runs to be merged could consist of a combination of initial $s_{1}$ sorted runs and $s_{12}$ sorted runs. The batched reading strategy can be easily modified so that composite chunklets within the $s_{12}$ runs, which have already been sorted on $o_{2}$, need not be internally sorted again as part of the merging.

\section{Discussions}

\section{A. Ascending/Descending Ordering}

In this section, we explain how to extend our proposed techniques to handle the general case where a sort order can consist of attributes to be sorted in a combination of ascending and descending orders. For a sort attribute $a$, let $a^{\prime}$ and $a^{\prime \prime}$ denote the ascending and descending ordering of $a$, respectively. We can treat $a^{\prime}$ and $a^{\prime \prime}$ as two different attributes in sort orders. For two sort orders $o_{1}$ and $o_{2}$, we refer to them as a reverse pair if (1) $o_{1}=o_{2}$ when ascending/descending orderings are ignored; and (2) for each attribute $a^{\prime}$ (resp. $a^{\prime \prime}$ ) in $o_{1}$, the corresponding attribute in $o_{2}$ is $a^{\prime \prime}$ (resp. $a^{\prime}$ ). Clearly, for a reverse pair, the result of one order can be easily converted into the result of the other by a backward scan of the sorted output.

We now revisit the four cases for $o_{1}$ and $o_{2}$ with the additional consideration of ascending/descending order. We only discuss cases where $o_{1}$ and $o_{2}$ satisfy one of the four cases if all their attributes were to be sorted in ascending order.

For cases 1 and 2, there must exist a longest pair of prefixes, $o_{11}$ and $o_{21}$, from $o_{1}$ and $o_{2}$, respectively, such that $\left(o_{11}, o_{21}\right)$ forms a reverse pair. By using a backward scan, we can treat $o_{11}$ and $o_{21}$ as a common prefix; thus, the result sharing technique is still applicable. For example, $o_{1}=\left(a^{\prime}, b^{\prime \prime}\right)$ and $o_{2}=\left(a^{\prime \prime}, b^{\prime}\right)$ still satisfy case 1 , while $o_{1}=\left(a^{\prime}, b^{\prime}\right)$ and $o_{2}=\left(a^{\prime \prime}, b^{\prime}\right)$ now satisfy case 2 .

For case 3 , cooperative sorting is still applicable. For a composite $s_{12}$ chunk, the ascending/descending orders can be handled by internal sorting. For a natural chunk, we generate it as usual with a sorted order $o_{12}$. To use this natural chunk as an initial run in $s_{2}$, its sort order should be $o_{21}$ (each tuple in the chunk has the same value for $\operatorname{attr} s\left(o_{22}\right)$ ). With a backward scan, $o_{12}$ and $o_{21}$ satisfy either case 1 or case 2. Therefore, we can easily convert the order of the natural chunk on-the-fly from $o_{12}$ to $o_{21}$ when it is merged for $s_{2}$.

Since case 4 is handled by reducing to case 3 , the discussion for case 4 is similar to case 3 . 


\section{B. Dynamic Optimization for Cases 3 and 4}

Recall that for cases 3 and 4, all the three sorting techniques (conventional sorting, result sharing, and cooperative sorting) are applicable. The choice of which technique to apply can actually be determined dynamically at run-time. Note that all the three techniques share a common step of generating initial $s_{1}$ sorted runs. After the initial $s_{1}$ runs have been computed, we have precise information on the number of distinct $o_{11}$ values, the number and sizes of $s_{12}$ chunks, and the sizes and distributions of the $s_{12}$ chunklets among the $s_{1}$ initial runs. With this information, we can more accurately determine the cost estimates of the three competing techniques and choose the most efficient technique to evaluate $s_{1}$ and $s_{2}$ at run-time.

\section{Vi. Optimization of Multiple Sortings}

In this section, we generalize our discussion of evaluating two sort operations to consider the evaluation of a collection of sort operations $S=\left\{s_{1}, s_{2}, \cdots, s_{k}\right\}, k \geq 2$, where each $s_{i}=\operatorname{sort}\left(T, o_{i}\right)$ is a sort operation on relation $T$ with sort order $o_{i}$. We first consider the extension of cooperative sort to handle more than two sort orders, and then explain how to optimize the evaluation of multiple sorts on a relation.

\section{A. K-way Cooperative Sort}

In Section IV, we develop cooperative sort to evaluate two sort operations $s_{1}$ and $s_{2}$. In general, given a collection of $k$ sort operations, a natural question to ask is whether it makes sense to generalize the binary cooperative sort to a $k$-way cooperative sort so that all $k$ sort operations can be efficiently evaluated. We refer to $k$ as the order of cooperative sort.

Given two sort orders $o_{i}$ and $o_{j}$, let $o_{i} \cdot o_{j}$ denote the sort order $o_{i}+\left(o_{j}-\operatorname{attr} s\left(o_{i}\right)\right)$ as discussed for case 4 in Section IV. Generalizing binary cooperative sort to $k$-way cooperative sort requires generating $k-1$ intermediate sort operations: $\left\{s_{2}^{\prime}, s_{3}^{\prime}, \cdots, s_{k}^{\prime}\right\}$, where the sort order $o_{i}^{\prime}$ associated with each $s_{i}^{\prime}$ is given by $o_{i}^{\prime}=o_{1} \cdot o_{2} \cdot o_{3} \cdot \ldots \cdot o_{i}$. Each $s_{i}^{\prime}$ corresponds to case 3 with sort orders $o_{i}^{\prime}$ and $o_{i}$.

$k$-way cooperative sort works as follows: $s_{1}$ is derived from $s_{2}^{\prime}$, and each $s_{i}, i>1$ is derived from $s_{i}^{\prime}$. The main idea of the extension is for all intermediate sorts to be derived from a single collection of initial runs that is sorted on $o_{k}^{\prime}$. Fortunately, the following analytical result based on our cost model shows that it is not necessary to consider $k$-way cooperative sort for $k>2$ (the proof can be found in [10]).

Theorem 6.1: For each query $Q$ involving multiple sorts on some relation, there exists an optimal query evaluation plan $P$ for $Q$ where the maximum order of cooperative sort used in $P$ is at most 2 .

Based on Theorem 6.1, we only consider binary cooperative sort in subsequent discussions and we simply use the term cooperative sort to refer to it.

\section{B. Multiple Sorting Optimization}

Given a collection $S$ of $k$ sort operations, there are many ways in which these operations can be ordered to exploit cooperative sort. In this section, we model this optimization problem as a graph problem. Given $S$, we construct a directed search graph $G(V, E)$, where $V=V_{a} \cup V_{b}, V_{a}$ represents the set of sort nodes and $V_{b}$ represents the set of cooperative sort operator nodes.

Each sort node $u \in V_{a}$ is associated with a sort order, denoted by $\operatorname{order}(u)$. For each sort operation $s=\operatorname{sort}(T, o)$ $\in S$, we create a sort node $u \in V_{a}$ and set $\operatorname{order}(u)$ to $o$. Each directed edge $(u, v)$ from sort node $u$ to sort node $v$ is associated with $\operatorname{cost}(u, v)$ equal to the cost of sorting $T$ that currently satisfies $\operatorname{order}(u)$ to satisfy $\operatorname{order}(v)$. There are two types of directed edges between sort nodes, corresponding to case 1 and case 2 in Section III.

For each pair of sort nodes $u$ and $v$ such that $\operatorname{order}(u)$ and $\operatorname{order}(v)$ satisfy case 3 or case 4, we create a new cooperative sort operator node $w \in V_{b}$ and associate $\operatorname{operand}(w)=(u, v)$. This node represents a cooperative sort operation from which $u$ and $v$ can be derived. From $w$, we add two directed edges: $(w, u)$ and $(w, v)$. Both $\operatorname{cost}(w, u)$ and $\operatorname{cost}(w, v)$ are labeled based on the cost model in Section IV.

Finally, an artificial root $\in V_{a}$ node is added to represent the relation $T$ without a particular order. We add an edge from root to each existing node $v$ in $V$, with $\operatorname{cost}($ root, $v$ ) equal to the cost of a conventional sorting operation.

Once the search graph has been constructed, the optimal solution can be found by computing the minimum directed Steiner tree of $G$. The terminal nodes of the Steiner tree are the set of sort nodes in $V_{a}$.

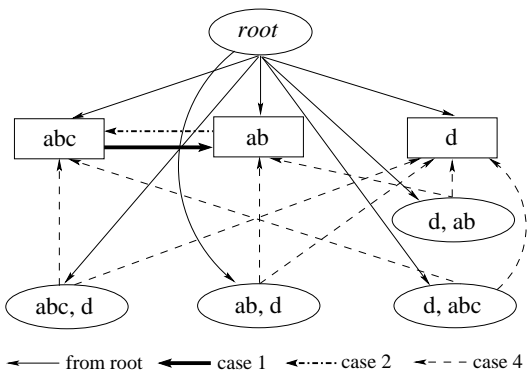

(a) The Sample Search Graph $G$

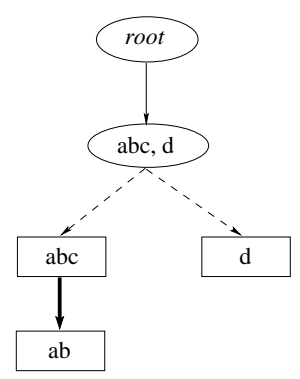

(b) Steiner Tree of $G$
Fig. 5. An Example of Multiple Sorting Optimization

Example 6.1 Consider three sort operations $\operatorname{sort}(T, a b)$, $\operatorname{sort}(T, a b c)$ and $\operatorname{sort}(T, d)$, where $a, b$, and $c$ are attributes of $T$. The search graph is depicted in Fig. 5(a), where the sort (resp. cooperative sort) nodes are represented by rectangles (resp. ellipses). The computed Steiner tree for this search graph is shown in Fig. 5(b). Based on the Steiner tree, a feasible evaluation plan is as follows: first perform cooperative sort between $\operatorname{sort}(T, a b c)$ and $\operatorname{sort}(T, d)$, and derive $\operatorname{sort}(T, a b)$ from $\operatorname{sort}(T, a b c)$.

Although finding the minimum directed Steiner tree is an NP-hard problem [11], applying a brute-force algorithm is actually adequate if $\left|V_{b}\right|$ is small. Basically, we enumerate every subset of $V_{b}$ to be used in the spanning tree and find one with the minimum cost. The complexity of finding the directed minimum spanning tree is $O\left(N^{2}\right)$ where $N$ is the number of nodes in the graph [12]. Hence, the total complexity of the algorithm is $O\left(2^{\left|V_{b}\right|}|V|^{2}\right)$. In our context, since $\left|V_{a}\right|$ is small 
and $\left|V_{b}\right| \leq\left|V_{a}\right|^{2}$ is also small, a brute-force solution is reasonable; otherwise, heuristic/approximation algorithms [13], [14] can be applied here.

\section{PeRformance Study}

We validated our ideas using a prototype built in PostgreSQL 8.3.5 [15]. All experiments were performed on a Dell workstation with a Quad-Core Intel Xeon $2.66 \mathrm{GHz}$ processor, $8 \mathrm{~GB}$ of memory, one 500G SATA disk and another 750GB SATA disk, running Linux 2.6.22. Both the operating system and PostgreSQL system are built on the 500GB disk, while the databases of PostgreSQL are stored on the 750GB disk.

This performance study focused on the effect of cooperative sort. In our implementation, the cooperative sort is integrated into PostgreSQL as a standard operator. It adopts k-way merge pattern and is capable of final merge optimization. For the purpose of fair comparison, we also converted the run merge pattern of the original sort operation in PostgreSQL from polyphase to k-way (We have evaluated the performance of our k-way merge implementation against PostgreSQL's polyphase merge scheme. Our results showed no significant differences between the two schemes. Readers may refer to [10] for details.). Moreover, we added a post-optimizer that implements the optimization techniques in Section VI. The post-optimizer receives an execution plan from the original query optimizer, exploits sharing and cooperation opportunities between sorts in a cost-based manner and, whenever possible, generates a cheaper plan enhanced with cooperative sorts. By switching on and off the post-optimizer, we can easily compare the cost of processing a query under the cooperative sort operation against that of the corresponding two independent sort operations.

\section{A. Micro-benchmark Test}

In this section, we use a micro-benchmark test to compare the performance of cooperative sort against two independent sort operations. We define a query template $Q$ :

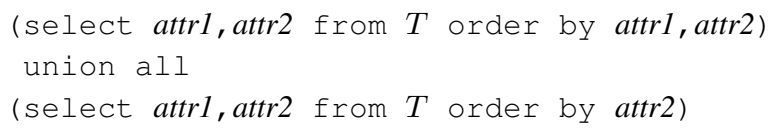

This template also serves to simulate two queries in a batch. The execution plan of $Q$ is an immediate result union of two sorts, $s_{1}$ and $s_{2}$, on the same relational table $T$. The sort orders of $s_{1}$ and $s_{2}$ are $(a t t r 1, a t t r 2)$ and $(a t t r 2)$ respectively and thus satisfy case 3 .

We generated six concrete queries with the above query template by using three different relations from the TPC-DS [16] benchmark for $T$ and two different scale factors (denoted by $S F$ ) to vary the size of $T$. The statistical information about three relations, along with their sort attributes, are shown in Table VII-A. The scale factor $S F$ values used are $40 \mathrm{~GB}$ and $100 \mathrm{~GB}$. Another experimental parameter that we varied is the available sorting memory dedicated to each sorting operation (denoted by $M$ ) with values ranging from $5 \mathrm{MB}$ to $200 \mathrm{MB}$. The sorting memory values are chosen such that at least half of them will result in a single run merge step.
We compare the performance of two basic evaluation techniques for sorting: the conventional technique of using two independent sorts (denoted by IS) and our proposed cooperative sort (denoted by CS). We also enable/disable the final merge optimization to study the combined effectiveness of this optimization with the basic techniques. We use CS-OPT and IS-OPT to denote the variants that have the optimization enabled, and CS and IS to denote the variants that have the optimization disabled.

Each total execution time reported refers to the total query evaluation time including the I/O cost of reading the sorted outputs of $s_{1}$ and $s_{2}$. Each query timing is measured with the query running alone in the database system; and the operating system is restarted between queries to clear the system cache.

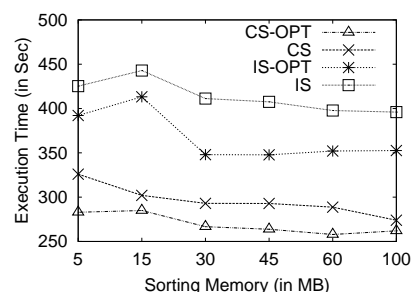

(a) web_sales, $S F 40$

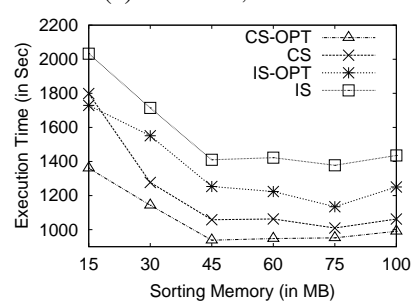

(c) store_sales, $S F 40$

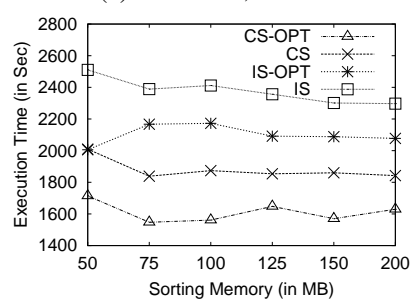

(e) catalog_sales, $S F 100$

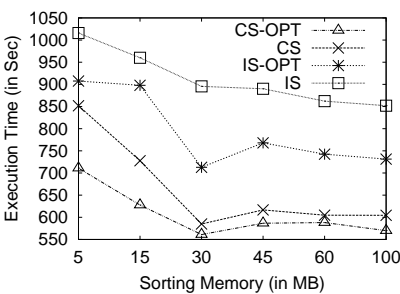

(b) catalog_sales, $S F 40$

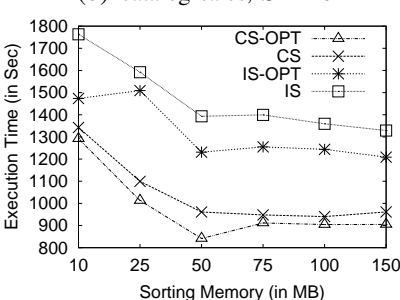

(d) web_sales, $S F 100$

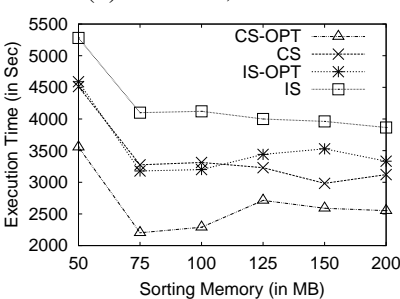

(f) store_sales, $S F 100$
Fig. 6. Performance Comparison on TPC-DS Dataset

1) General Results: Fig. 6 compares the performance of the four evaluation strategies as a function of the sorting memory size; the comparison for each query is shown on a separate graph. The detailed breakdown of the various cost components for CS and IS are shown in Table VII-A.1; only SF 40 dataset is shown due to space limitation, the complete results can be found in [10]. The meanings of these cost components are given in Table VII-A.1.

Due to space limitations, we shall not present detailed query-by-query analysis. Instead, we will summarize the more interesting findings here.

First, we observe that CS(-OPT) offers significant performance improvement over IS(-OPT) in most queries. The savings range from a few seconds to 1,033 seconds which is achieved by CS-OPT over IS-OPT for the query on store_sales with $M=50$ and $S F=100$ in Fig. 6. In terms of relative 
TABLE II

TESTED TPC-DS DATASET

\begin{tabular}{|c|c|c|c|c|}
\hline relation & attrl & attr2 & number of tuples (in million) & tuple size (in byte) \\
\hline web_sales & ws_item_sk & ws_sold_time_sk & $0.72 \times S F$ & 226 \\
\hline catalog_sales & CS_item_sk & CS_sold_time_sk & $1.44 \times S F$ & 226 \\
\hline store_sales & ss_item_sk & ss_sold_time_sk & $2.88 \times S F$ & 164 \\
\hline
\end{tabular}

TABLE III

The Component Costs of CS AND IS

\begin{tabular}{|c|c|c|}
\hline & notation & description \\
\hline \multirow{5}{*}{ CS } & $R F_{c s}\left(s_{12}\right)$ & $\begin{array}{l}\text { initial run formation cost for } s_{12} \\
\text { (i.e., creating initial } s_{1} \text { sorted runs) }\end{array}$ \\
\hline & $R M_{c s}\left(s_{12}\right)$ & $\begin{array}{l}\text { run merge cost for } s_{12} \\
\text { (i.e., creating } s_{12} \text { chunks) }\end{array}$ \\
\hline & $R M_{c s}\left(s_{2}\right)$ & $\begin{array}{c}\text { run merge cost for } s_{2} \\
\text { (i.e., merging } s_{12} \text { chunks to derive } s_{2} \text { ) }\end{array}$ \\
\hline & $S C_{c s}\left(s_{12}\right)$ & $\begin{array}{l}\text { cost of internal sorting to create } \\
\text { initial } s_{12} \text { runs from initial } s_{1} \text { runs }\end{array}$ \\
\hline & $S C_{c s}\left(s_{1}\right)$ & $\begin{array}{l}\text { cost of internal sorting during } \\
\text { the derivation of } s_{1} \text { output from } s_{12}\end{array}$ \\
\hline \multirow{4}{*}{ IS } & $R F_{i s}\left(s_{1}\right)$ & $\begin{array}{l}\text { initial run formation cost for } s_{1} \\
\text { (i.e., creating initial } s_{1} \text { sorted runs) }\end{array}$ \\
\hline & $R M_{i s}\left(s_{1}\right)$ & $\begin{array}{c}\text { run merge cost for } s_{1} \\
\text { (i.e., merging } s_{1} \text { sorted runs) }\end{array}$ \\
\hline & $R F_{i s}\left(s_{2}\right)$ & $\begin{array}{l}\text { initial run formation cost for } s_{2} \\
\text { (i.e., creating initial } s_{2} \text { sorted runs) }\end{array}$ \\
\hline & $R M_{i s}\left(s_{2}\right)$ & $\begin{array}{c}\text { run merge cost for } s_{2} \\
\text { (i.e., merging } s_{2} \text { sorted runs) }\end{array}$ \\
\hline
\end{tabular}

improvement, the average percentage improvement is around $25 \%$ and the highest improvement is $35 \%$ achieved by CS over IS for the query on catalog_sales with $M=30$ and $S F=40$.

Second, although operating on the same set of initial runs, the run merge phase of $s_{12}$ incurs a higher CPU cost than that of $s_{1}$ due to the additional tuple comparison steps. Note that $R M_{c s}\left(s_{12}\right)$ does not include the internal sorting cost $S C_{c s}\left(s_{12}\right)$. However, for all six queries, $R M_{c s}\left(s_{12}\right)$ is close to or even less than $R M_{i s}\left(s_{1}\right)$. This observation validates the I/O effectiveness and efficiency of our batched reading strategy.

Third, for all six queries, $R F_{c s}\left(s_{12}\right), R F_{i s}\left(s_{1}\right)$ and $R F_{i s}\left(s_{2}\right)$ are always more or less the same with any amount of sorting memory. This is due to the fact that during the initial run formation phase, the reading and writing of tuples to the disk files are interleaved and the cost of incurred random $\mathrm{I} / \mathrm{O}$ is independent of the size of the sorting memory. On the other hand, $R M_{c s}\left(s_{12}\right), R M_{c s}\left(s_{2}\right), R M_{i s}\left(s_{1}\right)$, and $R M_{i s}\left(s_{2}\right)$ all decrease when the sorting memory increases, as the larger sorting memory makes the run merging more I/O-efficient.

Finally, for all 6 tables, $S C_{c s}\left(s_{12}\right)$ and $S C_{c s}\left(s_{2}\right)$ increase along with the size of sorting memory. The reason is two-fold: on the one hand, the larger sorting memory means that more tuples will be combined into composite chunks/chunklets and more tuples need to be internally sorted; on the other hand, with the fixed total number of tuples, it is cheaper to independently sort many smaller composite chunks/chunklets than independently sort fewer larger composite chunks/chunklets, which is similar to the analysis of case 2 in Section III.

2) Effect of Result Sharing: As discussed at the beginning of Section IV-A, the result sharing technique (denoted by RS) can actually be applied to evaluate case 3. In this section, we compare the effectiveness of RS against CS for the six queries. Fig. 7 compares the performance of the query on web_sales with $S F$ 40; the comparison for other quries have similar trends and are omitted.

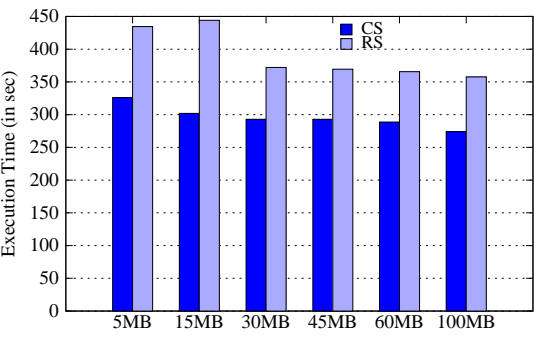

Fig. 7. Comparison of CS with RS on web_sales, $S F 40$

The results clearly demonstrate that CS significantly outperforms RS in all sorting memory settings. The performance of RS is just a little better than IS (see Fig. 6(a)).

\section{B. Synthetic Data}

We also utilize synthetic data to investigate the sensitivity of CS. We generated synthetic tables for the web_sales relation in TPC-DS benchmark using $S F=40$; each table has 28.8 million tuples. We run template query $Q$ defined in the previous section on the synthetic tables to compare the performance of CS and IS.

1) Varying Total Number of $s_{12}$ Chunks: Under CS, there will be $n$ initial runs for $s_{2}$ if $n$ chunks are formed by $s_{12}$. The purpose of this experiment is to learn how the total number of $s_{12}$ chunks will affect the run merge cost for $s_{2}$. We vary the number $n$ of distinct ws_item_sk (the $o_{11}$ ) values inside a web_sales table. Six values of $n$ are used: 15, 25, 50, 100, 150 and 200. A uniform distribution is used for the values of ws_item_sk. We fix the sort memory to $20 \mathrm{MB}$, so that even when $n$ is 200 the tuples with the same ws_item_sk value cannot fit in memory and thus will form a natural chunk. As a result, there will be totally $n$ natural chunks.

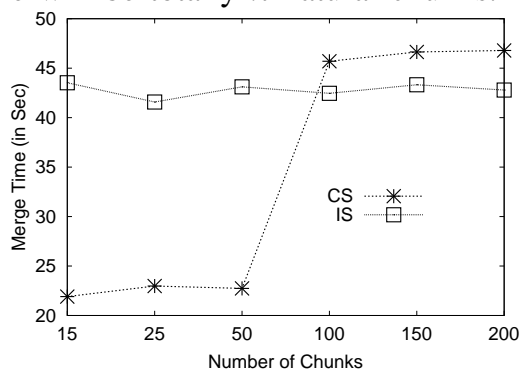

Fig. 8. Varying Total Number of $s_{12}$ Chunks

The experimental result is shown in Fig. 8. The y-axis denotes the run merge time for $s_{2}$. With 20MB sort memory, the merge order $F$ is 73 . Moreover, the number of initial runs to merge for $s_{2}$ under IS is 56. Therefore, with all the different $n$ values, the number of merge passes for IS on $s_{2}$ is always 1 and the merge costs are more or less the same. As for CS, the merge cost increases significantly when $n$ becomes larger than 73. This is because the number of merge passes changes from 1 to 2 . This confirms the expectation that the merge costs of CS remains largely unchanged with varying $n$ as long as the number of merge passes required stays the same. We also 
TABLE IV

COMponental Costs of SORTS In Micro-Benchmark TEST (IN SECONDS)

$\mathrm{CS}$

\begin{tabular}{|c|c|c|c|c|c|c|c|c|c|c|}
\hline & & & & CS & & & & & & \\
\hline & Memory & $R F_{c s}\left(s_{12}\right)$ & $R M_{c s}\left(s_{12}\right)$ & $R M_{c s}\left(s_{2}\right)$ & $S C_{C S}\left(s_{12}\right)$ & $S C_{c s}\left(s_{1}\right)$ & $R F_{i s}\left(s_{1}\right)$ & $R M_{i s}\left(s_{1}\right)$ & $R F_{i s}\left(s_{2}\right)$ & $R M_{i s}\left(s_{2}\right)$ \\
\hline \multirow{6}{*}{$\begin{array}{c}\text { web_sales } \\
S F 40\end{array}$} & $5 \mathrm{MB}$ & 129.25 & 70.29 & 59.15 & 3.45 & 22.98 & 127.39 & 70.90 & 128.71 & 54.43 \\
\hline & $15 \mathrm{MB}$ & 126.62 & 69.47 & 32.57 & 8.30 & 23.57 & 126.36 & 75.54 & 125.70 & 71.79 \\
\hline & $30 \mathrm{MB}$ & 129.62 & 58.64 & 28.12 & 11.47 & 23.80 & 126.52 & 60.05 & 126.24 & 53.60 \\
\hline & $45 \mathrm{MB}$ & 130.18 & 53.87 & 27.46 & 15.45 & 24.51 & 129.92 & 55.22 & 125.84 & 53.24 \\
\hline & $60 \mathrm{MB}$ & 126.27 & 47.89 & 28.81 & 18.41 & 24.85 & 126.23 & 50.96 & 129.36 & 47.61 \\
\hline & $100 \mathrm{MB}$ & 125.64 & 34.90 & 24.52 & 22.11 & 25.32 & 125.93 & 49.26 & 129.59 & 46.88 \\
\hline \multirow{6}{*}{$\begin{array}{c}\text { catalog_sales } \\
S F 40\end{array}$} & $5 \mathrm{MB}$ & 256.60 & 221.96 & 230.49 & 7.58 & 45.38 & 259.03 & 219.82 & 255.64 & 192.93 \\
\hline & $15 \mathrm{MB}$ & 260.75 & 229.75 & 91.48 & 16.65 & 46.29 & 263.36 & 188.90 & 254.87 & 164.14 \\
\hline & $30 \mathrm{MB}$ & 254.66 & 121.97 & 58.62 & 20.65 & 47.19 & 257.42 & 155.15 & 260.35 & 136.16 \\
\hline & $45 \mathrm{MB}$ & 258.27 & 149.05 & 55.25 & 25.48 & 47.21 & 260.98 & 150.07 & 258.29 & 132.78 \\
\hline & $60 \mathrm{MB}$ & 255.65 & 132.31 & 54.76 & 32.59 & 47.71 & 258.62 & 137.59 & 261.16 & 118.33 \\
\hline & $100 \mathrm{MB}$ & 262.61 & 118.78 & 51.89 & 40.62 & 48.43 & 261.75 & 126.01 & 269.65 & 106.86 \\
\hline \multirow{6}{*}{$\begin{array}{l}\text { store_sales } \\
\quad S F 40\end{array}$} & $15 \mathrm{MB}$ & 352.36 & 934.28 & 244.45 & 19.21 & 70.28 & 440.03 & 539.52 & 399.86 & 4992.84 \\
\hline & $30 \mathrm{MB}$ & 377.94 & 385.95 & 236.96 & 28.94 & 72.11 & 392.31 & 399.09 & 370.46 & 381.49 \\
\hline & $45 \mathrm{MB}$ & 362.83 & 195.38 & 224.75 & 39.27 & 72.91 & 351.04 & 277.77 & 358.31 & 259.73 \\
\hline & $60 \mathrm{MB}$ & 384.26 & 291.87 & 102.73 & 49.96 & 73.91 & 354.45 & 279.03 & 384.18 & 242.23 \\
\hline & $75 \mathrm{MB}$ & 377.61 & 243.56 & 93.21 & 64.51 & 75.17 & 380.68 & 256.74 & 360.36 & 217.99 \\
\hline & $100 \mathrm{MB}$ & 393.62 & 263.99 & 99.12 & 67.59 & 76.32 & 385.29 & 270.82 & 375.42 & 232.20 \\
\hline
\end{tabular}

notice that with the same number of merge passes, the merge cost of CS is always lower than that of IS, which is consistent with the observation in the micro-benchmark test.

2) Varying Number of Composite $s_{12}$ Chunks: In this experiment, we examine the contributions of internal sorting cost to the total CS cost. These internal sorts are applied to composite chunklets and chunks. We fix the total number $m$ of chunks generated and vary the number $n$ of composite chunks. We set the sort memory to $50 \mathrm{MB}$ and $m$ to 55 . Five values of $n$ are used: $0,13,27,42$ and 55 .

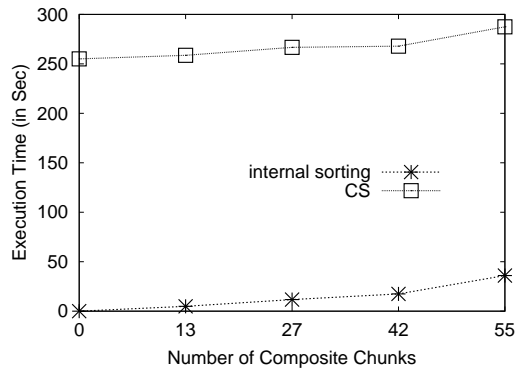

Fig. 9. Varying Number of Composite $s_{12}$ Chunks

Fig. 9 shows the internal sorting cost as well as the overall CS cost. As expected, the internal sorting cost increases along with the number of composite chunks. When all the 55 chunks become composite, this cost takes a non-trivial percentage of the total CS cost.

\section{Synthetic Database and Queries}

So far, we have evaluated cooperative sort for the basic scenario of processing 2 sort operations on different orders. In this section, we evaluate the effectiveness of cooperative sort on queries. We generate a synthetic database with three relations Employee(id, name, country_id, supervisor_id), Sales(employee_id, item_id, quantity, profit) and Item(id, name). Employee records the information of employed salesperson and has 10 million 32-byte tuples; Sales records the transactions and has 50 million 12-byte tuples; Item records the products on sale and has 10 million 24-byte tuples.

We define two queries on this database:

Q1: Find the name of each salesperson and its supervisor.
Q2: Find each salesperson that has sold more than 1000 units of a product in a single transaction or his supervisor has done so.

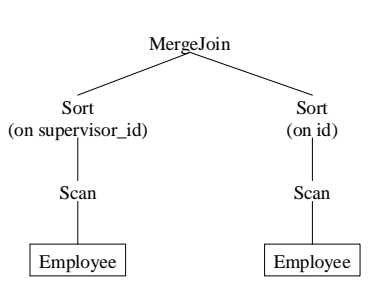

(a) Q1

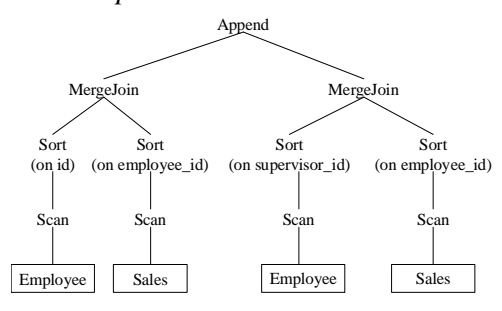

(b) Q2
Fig. 10. The Optimal Plans for Q1 and Q2

With 50MB sort memory, the optimal plans generated by PostgreSQL for these two queries are shown in Fig. 10.

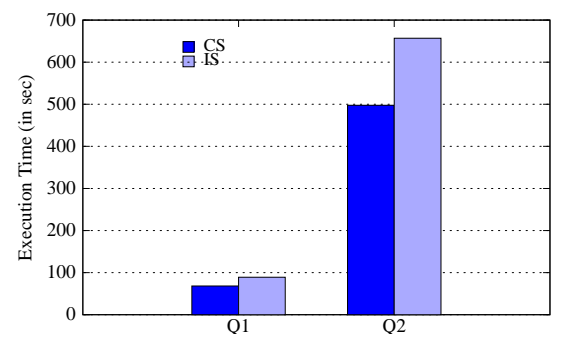

Fig. 11. Query Execution Times of Q1 and Q2

We run Q1 and Q2 with both CS and IS. For Q2, one redundant sort on sales is also skipped by the post-optimizer, which contributes about 90 seconds' saving. The overall query execution times are shown in Fig. 11. The results clearly show that both queries can be processed in lesser time under CS.

\section{Impact on Optimizer Search Space}

In this experiment, we study the potential benefit of enriching the optimizer search space with cooperative sort. We make use of the synthetic database and Q1 from Section VIIC. In PostgreSQL, each sorting and hashing operation has a dedicated operator memory. We vary this operator memory and compare various execution plans for Q1: Hybrid Hash Join (HHJ), Sort Merge Join (SMJ) and Sort Merge join with Cooperative Sort (SMJ-CS). As shown in Fig. 12, the original optimizer of PostgreSQL generates either SMJ or HHJ as the optimal plan for Q1. We can find that with 10MB operator 
memory, SMJ-CS beats the optimal HHJ, which indicates that a cooperative-sort-aware query optimizer is promising.

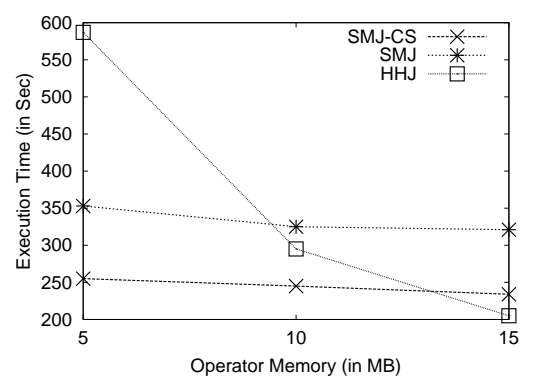

Fig. 12. Optimization Potential of CS

\section{RELATED WORK}

Sorting is one of the most extensively studied problems in computing. Knuth's classical text [1] provides extensive coverage of the fundamentals of sorting, including both replacement selection for run formation and run merge patterns.

Larson [17] introduced a cache-aware replacement selection that works for various length keys. There are also lots of techniques to speed up the run merge phase [4], [5], [6], focusing on how to improve $\mathrm{I} / \mathrm{O}$ performance during the merge phase because this phase is typically I/O bound. These techniques are however complementary to our batched reading strategy, which relies more on the pre-collected knowledge about input data distribution. Our current implementation only applies simple forecasting technique to the type- 3 reads. But it is possible to incorporate other optimization techniques like double buffering [1], read-ahead [5], etc. Much research has been done on adaptive sorting [18] exploiting near-sortedness. Graefe's survey [19] discussed how sorting is implemented in database systems with many tricks and optimizations.

Simmen et.al [20] described how to determine the ordering propagation from the inputs to the outputs of joins, based on functional dependencies and selection conditions. Their work was followed and extended by [21], [22], [23], which are all independent and complementary to our work.

In [8], Sudarshan et. al observed that the order requirements of operators are often partially satisfied by the inputs. They proposed to maximize the benefit of such partial sort order by modifying the standard replacement selection algorithm and improving the selection of interesting orders. We instead consider the opportunity of partial sorting sharing between two distinct sort operations (case 2 in Section III). A similar idea to partial sorting was considered previously in [9] for the CUBE operator, which computes group-bys corresponding to all possible combinations of a list of attributes. Consider two group-bys $B=\left\{a_{1}, a_{2}, \ldots, a_{j}\right\}$ and $S=\left\{a_{1}, a_{2}, \ldots, a_{l-1}\right.$, $\left.a_{l+1}, \ldots, a_{j}\right\}$. With sort-based aggregation, the result of $B$ can be viewed as a concatenation of one or more partitions and the result of $S$ is the union of independently computing aggregation within each partition.

Finally, there have been a few previous works on optimizing multiple scans on the same table, such as MAPLE [7] and cooperative scan [24], etc.

\section{CONCLUSION}

In this paper, we have examined the problem of sorting a relational table on multiple sort orders. Such collections of sortings are common in many applications. We have identified several cases in which the (partial) work done in sorting a table on a particular order can be re-used for a subsequent sort of the same table on a different order. We proposed the cooperative sort technique to efficiently handle sorting of a table on two orders. We also proposed a post-optimizer to exploit cooperative sort in a traditional query evaluation plan. We have implemented our techniques in PostgreSQL, and our extensive performance study indicated a performance gain of upto $35 \%$ over the naive strategy of processing each sort independently.

Acknowledgements This research is supported in part by NUS Grant R-252-000-271-112.

\section{REFERENCES}

[1] D. E. Knuth, The art of computer programming, volume 3: (2nd ed.) sorting and searching. Addison-Wesley, 1998.

[2] V. Pai and P. Varman, "Prefetching with multiple disks for external mergesort: simulation and analysis," in ICDE, 1992.

[3] B. Salzberg, "Merging sorted runs using large main memory," Acta Informatica, vol. 27, no. 3, 1989.

[4] W. Zhang and P. Larson, "Dynamic memory adjustment for external mergesort," in $V L D B, 1997$.

[5] W. Zhang and P.-A. Larson, "Buffering and read-ahead strategies for external mergesort," in $V L D B, 1998$.

[6] L. Zheng and P. Larson, "Speeding up external mergesort," IEEE Transactions on Knowledge and Data Engineering, vol. 8, no. 2, 1996.

[7] Y. Cao, G. C. Das, C.-Y. Chan, and K.-L. Tan, "Optimizing complex queries with multiple relation instances," in SIGMOD, 2008.

[8] R. Guravannavar and S. Sudarshan, "Reducing order enforcement cost in complex query plans," in ICDE, 2007.

[9] S. Agarwal, R. Agrawal, P. Deshpande, A. Gupta, J. F. Naughton, R. Ramakrishnan, and S. Sarawagi, "On the computation of multidimensional aggregates," in $V L D B, 1996$.

[10] Y. Cao, R. Bramandia, C.-Y. Chan, and K.-L. Tan, "Optimized query evaluation using cooperative sorts, technical report," in www.comp.nus.edu.sg/ caoyu/icde-TR.pdf.

[11] R. M. Karp, "Reducibility among combinatorial problems," in Complexity of Computer Computations. Plenum Press, 1972.

[12] L. Georgiadis, "Arborescence optimization problems solvable by edmonds' algorithm," Theor. Comput. Sci., vol. 301, no. 1-3, 2003.

[13] M.-I. Hsieh, E. H.-K. Wu, and M.-F. Tsai, "Fasterdsp: A faster approximation algorithm for directed steiner tree problem," J. Inf. Sci. Eng., vol. 22 , no. 6,2006

[14] M. Charikar, C. Chekuri, Z. Dai, A. Goel, S. Guha, and M. Li, "Approximation algorithms for directed steiner problems," in Journal of Algorithms, 1999.

[15] "Postgresql Offical Website," http://www.postgresql.org/.

[16] “TPC BENCHMARK Decision Support," http://www.tpc.org/tpcds/.

[17] P.-A. Larson, "External sorting: Run formation revisited," IEEE Transactions on Knowledge and Data Engineering, vol. 15, no. 4, 2003.

[18] V. Estivill-Castro and D. Wood, "A survey of adaptive sorting algorithms," ACM Computing Surveys (CSUR), vol. 24, no. 4, 1992.

[19] G. Graefe, "Implementing sorting in database systems," ACM Comput. Surv., vol. 38, no. 3, 2006.

[20] D. Simmen, E. Shekita, and T. Malkemus, "Fundamental techniques for order optimization," in SIGMOD, 1996.

[21] T. Neumann and G. Moerkotte, "A combined framework for grouping and order optimization," in $V L D B, 2004$.

[22] X. Wang and M. Cherniack, "Avoiding sorting and grouping in processing queries," in $V L D B, 2003$.

[23] T. Neumann and G. Moerkotte, "An efficient framework for order optimization," in ICDE, 2004.

[24] M. Zukowski, S. Héman, N. Nes, and P. A. Boncz, "Cooperative scans: Dynamic bandwidth sharing in a dbms," in VLDB, 2007. 